\title{
ON MARTINET'S SINGULAR SYMPLECTIC STRUCTURES
}

\author{
W. DOMITRZ and S. JANECZKO \\ Institute of Mathematics, Warsaw University of Technology \\ Pl. Politechniki 1, 00-661 Warszawa, Poland
}

Introduction. Let $V$ be a stratified subspace of $R^{N}$. We call it symplectic if there exists a differential 2-form $\omega$ on $R^{N}$ such that the restriction of $\omega$ to each stratum is a symplectic form. In the Marsden-Weinstein singular reduction theory these spaces were studied by several authors $[5,4,9,1]$. In this paper we classify the symplectic spaces modelled on the so-called symplectic flag $S$. First we prove the corresponding Darboux theorem and then we show that the only reasonable symplectic structures on $S$ are those with underlying Martinet's singular symplectic structure of type $\Sigma_{2,0}$. Finally we find the normal form for this structure and show the similar result for an example of a stratified symplectic space with singular boundary of the maximal stratum.

1. Singular symplectic spaces. A stratified differential space with each stratum being a symplectic manifold is called a stratified symplectic space. This notion was introduced in [9] (see also [4]) in the context of standard symplectic reduction. For our purpose, in the first step we need embedded symplectic spaces.

Defintion 1.1. Let $S$ be a stratified subset of $R^{N}$ with each stratum $S_{i}$ (even dimensional) endowed with a symplectic structure $\omega_{S_{i}}$. We assume that there exists a closed two-form $\omega$ on $R^{N}$ such that $\left.\omega\right|_{S_{i}}=\omega_{S_{i}}$. Then the pair $(S, \omega)$ is called a singular symplectic space.

A representative model of a singular symplectic space is a disjoint union of semialgebraic sets. We consider the following elementary symplectic flag:

$$
\begin{gathered}
S=S_{2 n} \cup S_{2 n-2} \subset R^{2 n} ; \\
S_{2 n}=\left\{(x, y) \in R^{2 n}: x_{1}>0\right\}, \quad S_{2 n-2}=\left\{(x, y) \in R^{2 n}: x_{1}=0, y_{1}=0\right\}
\end{gathered}
$$

endowed with a symplectic structure $\omega$. By $\iota_{k}: S_{i} \rightarrow R^{N}$ we denote the canonical inclusions of $S_{2 n-k}$. Here $S_{2 n-1}=\left\{x \in R^{2 n}: x_{1}=0\right\}$.

1991 Mathematics Subject Classification: 57R45, 53B21.

The paper is in final form and no version of it will be published elsewhere. 
ExAmPLE 1.1. Let $V \subset(M, \omega)$ be an algebraic hypersurface. Let $\mathcal{X}_{V}$ be its Whitney stratification. By $V^{d}$ we denote an element of $\mathcal{X}_{V}, V^{d} \in \mathcal{X}_{V}$, of dimension $d$. We say $V$ is a coisotropic hypersurface if and only if each stratum of $\mathcal{X}_{V}$ is a coisotropic or an isotropic submanifold of $(M, \omega)$. We easily see that a typical hypersurface $V$ defined by the polynomial equation $F(p)=0$ is not coisotropic. As an example let us consider the cusp-edge surface $V$ in $R^{2 n}$ endowed with a symplectic form $\omega$ in general position with respect to $V$. In this case $\left.\omega\right|_{\operatorname{Sing} V}$ is a symplectic form. It is shown in $[2]$ that $(V, \omega)$ is diffeomorphic to $\left(\left\{x_{1}^{3}-y_{1}^{2}=0\right\}, \sum_{i=1}^{n} d x_{i} \wedge d y_{i}\right)$ and the reduced symplectic space of $V$ - Sing $V$ is isomorphic to the singular edge of $V$ (cf. [4]).

We conjecture that if $\operatorname{Sing} V$ is a coisotropic submanifold of $\left(R^{2 n}, \omega\right)$, then $(V, \omega)$ is diffeomorphic to $\left(\left\{x_{1}^{3}-x_{2}^{2}=0\right\}, \sum_{i=1}^{n} d x_{i} \wedge d y_{i}\right)$. Let $\Phi: R^{2 n-1} \rightarrow R^{2 n}$ be the parameterization of $\left\{x_{1}^{3}-x_{2}^{2}=0\right\}$,

$$
\Phi\left(s, y_{1}, y_{2}, x_{3}, y_{3}, \ldots, x_{n}, y_{n}\right)=\left(s^{2}, y_{1}, s^{3}, y_{2}, x_{3}, y_{3}, \ldots, x_{n}, y_{n}\right) .
$$

Then

$$
\Phi^{*} \omega=d s \wedge d\left(3 s^{2} y_{2}+2 s y_{1}\right)+\sum_{i=3}^{n} d x_{i} \wedge d y_{i} .
$$

Let $\pi: R^{2 n-1} \rightarrow R^{2 n-2}$ be the mapping

$$
\pi\left(s, y_{1}, y_{2}, x_{3}, y_{3}, \ldots, x_{n}, y_{n}\right)=\left(s, 3 s^{2} y_{2}+2 s y_{1}, x_{3}, y_{3}, \ldots, x_{n}, y_{n}\right) .
$$

Let $S$ be the image of $\pi$. Then

$$
S=\left\{(x, y) \in R^{2 n-2}: x_{1} \neq 0\right\} \cup\left\{(x, y) \in R^{2 n-2}: x_{1}=0, x_{2}=0\right\}
$$

and

$$
\pi^{*}\left(\sum_{i=1}^{n-1} d x_{i} \wedge d y_{i}\right)=\Phi^{*} \omega
$$

The reduced space $S$ endowed with the Darboux form on $R^{2 n-2}$ is a singular symplectic space.

Now we have a natural extension problem: let $\tilde{\omega}$ be a symplectic form on $S_{2 n-2}$, we ask for the existence of the closed two-form on $R^{N}$ such that $\left.\omega\right|_{S_{2 n-2}}=\tilde{\omega}$ and $\left.\omega\right|_{S_{2 n}}$ is symplectic. The first step in approaching this problem is to classify singular symplectic spaces $(S, \omega)$, where $\omega$ provides a symplectic structure on $R^{2 n}$.

By $G_{S}$ we denote the group of germs of diffeomorphisms $\left(R^{2 n}, 0\right) \rightarrow\left(R^{2 n}, 0\right)$ preserving $S$, i.e. if $\Phi \in G_{S}$ then $\Phi\left(S_{2 n}\right) \subset S_{2 n}$, and $\Phi\left(S_{2 n-2}\right) \subset S_{2 n-2}$.

Let $\Phi \in G_{S}$. Then using the standard setting of singularity theory (cf. [7]) we have

$$
\Phi\left(x_{1}, y_{1}, \ldots, x_{n}, y_{n}\right)=\left(x_{1} \phi_{1}(x, y), x_{1} \phi_{12}(x, y)+y_{1} \phi_{22}(x, y), \phi_{3}(x, y), \ldots, \phi_{2 n}(x, y)\right),
$$

where $\phi_{1}, \phi_{12}, \phi_{22}, \phi_{3}, \ldots, \phi_{2 n}$ are smooth germs of functions on $\left(R^{2 n}, 0\right)$.

Let $\omega_{1}, \omega_{2}$ be two symplectic structures on $S$ (closed two-forms on $\left.\left(R^{2 n}, 0\right)\right)$.

Definition 1.2. We say that $\omega_{1}$ and $\omega_{2}$ are $S$-equivalent $\left(\omega_{1} \sim_{S} \omega_{2}\right)$ if and only if there exists $\Phi \in G_{S}$ such that $\Phi^{*} \omega_{1}=\omega_{2}$. 
Theorem 1.1 (Darboux form). Let $\omega$ be a symplectic structure on $S$. Assume $\omega$ is a symplectic form on $R^{2 n}$. Then $\omega$ is $S$-equivalent to the Darboux form:

$$
\omega \sim_{S} \sum_{i=1}^{n} d x_{i} \wedge d y_{i}
$$

Pro of. We take the homotopy (cf. [8]) $\omega_{t}=t \omega_{1}+(1-t) \omega_{0}, t \in[0,1]$. One can check that $\omega_{t}$ is a nondegenerate form for every $t \in[0,1]$. We seek for a smooth family $t \rightarrow \Phi_{t}$ such that

$$
\Phi_{t}^{*} \omega_{t}=\omega_{0}, \quad \Phi_{0}=i d_{R^{2 n}}
$$

Differentiating (1) we have

$$
L_{V_{t}} \omega_{t}+\omega_{1}-\omega_{0}=0,
$$

where $L_{V_{t}}$ is the Lie derivative along the vector field $V_{t}$ generated by the flow $\Phi_{t}$. But

$$
\left.\left.\left.L_{V_{t}} \omega_{t}=d\left(V_{t}\right\rfloor \omega_{t}\right)+V_{t}\right\rfloor d \omega_{t}=d\left(V_{t}\right\rfloor \omega_{t}\right) .
$$

We have $d\left(\omega_{0}-\omega_{1}\right)=0$ and $\iota_{2 n-1}^{*}\left(\omega_{0}-\omega_{1}\right)=0$. So by the relative Poincaré Lemma (see e.g. [11]) there exists a one-form $\alpha$ such that $d \alpha=\omega_{0}-\omega_{1}$ and $\alpha$ vanishes on $S_{2 n-1}$. Thus we have

$$
\left.V_{t}\right\rfloor \omega_{t}=\alpha \quad \text { and }\left.\quad \alpha\right|_{(x, y)}=0 \quad \text { for every }(x, y) \in S_{2 n-1} .
$$

Because $\omega_{t}$ is a nondegenerate form, (2) is always solvable with respect to $V_{t}$ and moreover $V_{t}(x, y)=0$ for every $(x, y) \in S_{2 n-1}$. We deduce $\Phi_{t}$ exists, $\Phi_{t} \in G_{S}$ and by compactness of the interval $[0,1]$ we have $\Phi^{*} \omega_{1}=\omega_{0}$.

2. Martinet's singular symplectic spaces. Before we pass to the more detailed analysis of the degenerate case we recall the basic results on the standard classification of singularities of differential forms [6].

Let $\omega$ be a germ of a closed two-form on $R^{2 n}$ at zero. We denote

$$
\Sigma_{k}(\omega)=\left\{x \in R^{2 n}: \operatorname{rank} \omega(x)=2 n-k\right\}, \quad k \text { is even. }
$$

Let $\omega^{n}=f \Omega$, where $\Omega$ is the volume form on $R^{2 n}$.

(i) If $f(0) \neq 0$ then $\omega$ is a symplectic form (according to the standard notation denoted by $\left.\Sigma_{0}\right)$ and by the Darboux theorem we obtain

$$
\omega=\sum_{i=1}^{n} d x_{i} \wedge d y_{i}
$$

in local coordinates around zero.

(ii) Next we assume $f(0)=0$ while $(d f)(0) \neq 0$. We have $\Sigma_{2}(\omega)=\{f=0\}$ and let $\iota: \Sigma_{2}(\omega) \rightarrow R^{2 n}$ be the inclusion. If $\iota^{*} \omega^{n-1}(0) \neq 0$ then in local coordinates

$$
\omega=x_{1} d x_{1} \wedge d y_{1}+\sum_{i=2}^{n} d x_{i} \wedge d y_{i}
$$

and this type of singular form $\omega$ is denoted by $\Sigma_{2,0}$ (and called Martinet's singular form).

Both types of forms $\Sigma_{0}, \Sigma_{2,0}$ are locally stable (see [6]) and this is why we use them in what follows. 
Proposition 2.1. Let $\omega$ be a symplectic structure on $S$. Assume $f(0)=0$ and $d f_{0} \neq 0$ (stability conditions), then $\omega$ is a singular form of type $\Sigma_{2,0}$ at zero, i.e. $\omega$ belongs to the standard orbit of (ii) (4).

Remark 2.1. We see that the symplectic form $\omega$ on $S$ may be very singular in general. The singular set of $\omega$ is not visible from $S$ (see Fig. 1). The above proposition says that the typical symplectic forms on $S$ can only have $\Sigma_{2,0}$ or $\Sigma_{0}$ type singularities in the ambient space. Thus the two remaining stable cases $\Sigma_{2,2,0}$ are naturally excluded from our approach (cf. [3]).

Proof of Proposition 2.1. We see that $\omega$ is a symplectic form on $S_{2 n-2}$. Let

$$
\widetilde{S}=\Sigma_{2}(\omega)=\{f=0\},
$$

where $\omega^{n}=f \Omega$ and $\Omega$ is the standard volume form on $R^{2 n}$. We have $T_{0} \widetilde{S}=T_{0} S_{2 n-1}$, because $\omega$ is symplectic on $S_{2 n}$. $S_{2 n-2} \subset S_{2 n-1}$ so $T_{0} S_{2 n-2} \subset T_{0} S_{2 n-1}$ and $T_{0} S_{2 n-2} \subset T_{0} \widetilde{S}$. By assumption $\iota_{2 n-2}^{*} \omega$ is symplectic. Thus $\left(\iota_{2 n-2}^{*} \omega\right)^{n-1} \neq 0$ and this implies $\left(\iota^{*} \omega\right)^{n-1} \neq$ 0 , where $\iota: \widetilde{S} \rightarrow R^{2 n}$ is the embedding of $\widetilde{S}$.

LEMMA 2.1. By means of a diffeomorphism $\Phi \in G_{S}$ of the form

$$
\Phi(x, y)=\left(\phi(x, y), x_{2}, \ldots, x_{n}, y_{1}, \ldots, y_{n}\right)
$$

one can reduce $f$ to the following normal form:

$$
f\left(x_{1}, y_{1}, \ldots, x_{n}, y_{n}\right)= \pm\left(x_{1}-\psi\left(y_{1}, x_{2}, y_{2}, \ldots, x_{n}, y_{n}\right)\right) .
$$

DEFINITION 2.1. We say that $\psi_{1}, \psi_{2}$ are contact equivalent if and only if there exists a diffeomorphism $\Phi:\left(R^{2 n-1}, 0\right) \rightarrow\left(R^{2 n-1}, 0\right)$ and a smooth function-germ $g:\left(R^{2 n-1}, 0\right) \rightarrow$ $R, g(0) \neq 0$, such that

$$
\psi_{1}=g \cdot\left(\psi_{2} \circ \Phi\right) .
$$

Let $\omega_{1}, \omega_{2}$ be two symplectic forms on $S$. Let $f_{1}, f_{2}$ define their corresponding singular hypersurfaces, $\omega_{1}^{n}=f_{1} \Omega$ and $\omega_{2}^{n}=f_{2} \Omega$ and $\psi_{1}, \psi_{2}$ are as in Lemma 2.1. By straightforward check we obtain the following

Proposition 2.2. If $\omega_{1}$ and $\omega_{2}$ are $S$-equivalent then $\psi_{1}$ and $\psi_{2}$ are contact equivalent.

Let $\omega$ be a symplectic form on $S, \omega^{n}=f \Omega, f(0)=0$ and $d f_{0} \neq 0$. We see that $\frac{\partial f}{\partial x_{i}}(0)=0$ and $\frac{\partial f}{\partial y_{j}}(0)=0$ for $i=2, \ldots, n, j=1, \ldots, n$, so $\frac{\partial f}{\partial x_{1}}(0) \neq 0$. Thus

$$
d f \wedge d y_{1} \wedge d x_{2} \wedge d y_{2} \wedge \ldots \wedge d x_{n} \wedge d y_{n}(0) \neq 0,
$$

so $\left\{y_{1}, x_{2}, y_{2}, \ldots, x_{n}, y_{n}\right\}$ defines a coordinate system on

$$
\widetilde{S}=\{f=0\} \text {. }
$$

Before we formulate the main theorem concerning the normal form of $\omega$ we need some necessary facts $([6])$.

LEMMA 2.2. Let $\tau$ be a $k$-form on $R^{n}$ satisfying

$$
\left.\left.\frac{\partial}{\partial x_{1}}\right\rfloor \tau=0, \quad \frac{\partial}{\partial x_{1}}\right\rfloor d \tau=0 .
$$




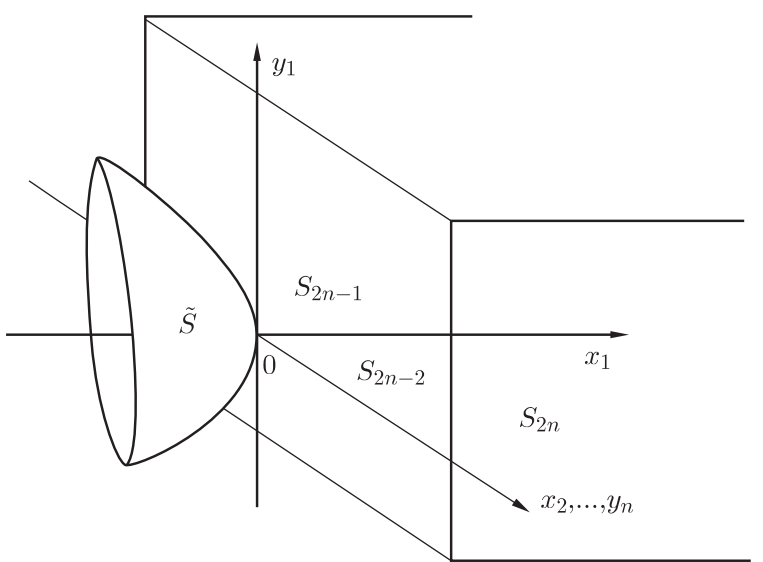

Fig. 1

Then $\tau=\pi^{*} \iota^{*} \tau$, where

$$
\begin{gathered}
\pi: R^{n} \rightarrow\left\{x_{1}=0\right\}, \quad \pi\left(x_{1}, x_{2}, \ldots, x_{n}\right)=\left(0, x_{2}, \ldots, x_{n}\right), \\
\iota:\left\{x_{1}=0\right\} \rightarrow R^{n}, \quad \iota\left(x_{2}, \ldots, x_{n}\right)=\left(0, x_{2}, \ldots, x_{n}\right) .
\end{gathered}
$$

LEMMA 2.3. Let $\tau$ be a $k$-form on $R^{n}$ satisfying

$$
\left.\left.\frac{\partial}{\partial x_{1}}\right\rfloor \tau=0, \quad \frac{\partial}{\partial x_{1}}\right\rfloor d \tau=\varphi \tau,
$$

where $\varphi$ is a smooth function on $R^{n}$. Then

$$
\tau=\zeta \pi^{*} \iota^{*} \tau,
$$

where $\zeta$ is a smooth function on $R^{n}$, and $\left.\zeta\right|_{\left\{x_{1}=0\right\}}=1$.

It is easy to prove the following lemmas.

LEMMA 2.4. Let $\alpha$ be a germ of a closed ( $n-1)$-form on $R^{n}$ at 0 satisfying the following conditions:

1. $\alpha_{0} \neq 0$,

2. a germ of a vector field $X$ at 0 such that $X\rfloor \alpha=0$ and $X(0) \neq 0$ meets $\left\{x_{1}=0\right\}$ transversally at 0 .

Then there exists a germ of diffeomorphism $\Phi:\left(R^{n}, 0\right) \rightarrow\left(R^{n}, 0\right)$, which preserves $\left\{x_{1}=0\right\}$ and

$$
\Phi^{*} \alpha=d x_{2} \wedge \ldots \wedge d x_{n}
$$

where $\left(x_{1}, \ldots, x_{n}\right)$ is a coordinate system on $R^{n}$.

LEMMA 2.5. Let $\alpha$ be a germ of a 1-form on $R^{2 k+1}$ at 0 satisfying the following conditions:

1. $\alpha \wedge(d \alpha)_{0}^{k} \neq 0$,

2. a germ of a vector field $X$ at 0 such that

$$
X\rfloor \alpha \wedge(d \alpha)^{k}=(d \alpha)^{k}
$$


meets $\{z=0\}$ transversally at 0 ,

3. $\iota^{*} \alpha_{0} \neq 0$, where $\iota:\{z=0\} \hookrightarrow R^{2 k+1}$ is the canonical inclusion.

Then there exists a germ of diffeomorphism $\Phi:\left(R^{2 k+1}, 0\right) \rightarrow\left(R^{2 k+1}, 0\right)$, which preserves $\{z=0\}$ and

$$
\Phi^{*} \alpha=d z+d y_{1}+\sum_{i=1}^{k} x_{i} d y_{i}
$$

where $\left(z, x_{1}, \ldots, x_{n}, y_{1}, \ldots, y_{n}\right)$ is a coordinate system on $R^{n}$.

Now we prove the main theorem obtaining the normal form (with moduli) of the symplectic structure on $S$. The geometrical contents of this theorem is illustrated in Fig. 1.

TheOREM 2.1. Let $\omega$ be a symplectic structure on $S$. Assume $f(0)=0$ and $d f_{0} \neq 0$. Then $\omega$ is $S$-equivalent to the form

$$
\begin{aligned}
\left(x_{1}-\psi\left(x_{2}, \ldots, x_{n}, y_{1}, \ldots, y_{n}\right)\right) d\left(x_{1}-\psi\left(x_{2}, \ldots, x_{n}, y_{1}, \ldots, y_{n}\right)\right) & \wedge d y_{1} \\
& +\sum_{i=2}^{n} d x_{i} \wedge d y_{i}
\end{aligned}
$$

where $\psi$ is a germ at 0 of a smooth function, $\psi(0)=0, \frac{\partial \psi}{\partial x_{i}}(0)=0, i=2, \ldots, n$, $\frac{\partial \psi}{\partial y_{i}}(0)=0, i=1, \ldots, n$.

Proof. By Lemma 2.1 we have $f= \pm\left(x_{1}-q\right)$, where $q$ does not depend on $x_{1}$. We are searching for a 1-form $\alpha$ satisfying the following conditions:

1. $d \alpha=\omega$,

2. $\iota^{*} \alpha \wedge\left(d \iota^{*} \alpha\right)_{0}^{n-1} \neq 0$, where $\iota: \tilde{S} \hookrightarrow R^{2 n}$ is the canonical inclusion,

3. $\tilde{\iota}^{*} \alpha_{0} \neq 0$, where $\tilde{\iota}: \tilde{S} \cap\left\{y_{1}=0\right\} \hookrightarrow R^{2 n}$ is the canonical inclusion.

$\omega$ is closed, then there exists a 1 -form $\alpha$ such that $d \alpha=\omega$. If $\alpha$ fails to satisfy condition 3 then we replace it by the 1 -form $\alpha+d y_{2}$, which satisfies conditions 1 and 3 .

Since $S_{2 n-2}$ is symplectic and $T_{0} S_{2 n-2}=T_{0}\left(\tilde{S} \cap\left\{y_{1}=0\right\}\right)$, we have $\left(\tilde{\iota}^{*} d \alpha\right)_{0}^{n-1}=$ $\left(\tilde{\iota}^{*} \omega\right)_{0}^{n-1} \neq 0$. Hence by Lemma 2.4 , we obtain

$$
\delta^{*} \iota^{*}(d \alpha)^{n-1}=d x_{2} \wedge \ldots \wedge d x_{n} \wedge d y_{1} \wedge \ldots \wedge d y_{n},
$$

where $\delta:(\tilde{S}, 0) \rightarrow(\tilde{S}, 0)$ is a diffeomorphism which preserves $\tilde{S} \cap\left\{y_{1}=0\right\}$. Therefore

$$
\iota^{*} d\left(\Delta^{*} \alpha\right)^{n-1}=d x_{2} \wedge \ldots \wedge d x_{n} \wedge d y_{1} \wedge \ldots \wedge d y_{n},
$$

where $\Delta \in G_{S}$ and

$$
\Delta(x, y)=\left(x_{1}, \delta\left(x_{2}, \ldots, x_{n}, y_{1}, \ldots, y_{n}\right)\right) .
$$

If $\Delta^{*} \alpha$ fails to satisfy condition 2 , then we replace it by the 1 -form $\Delta^{*} \alpha+d y_{1}$, which satifies all the conditions.

From condition 2 it follows that a vector field $X$ which satisfies the conditions

$$
X\rfloor \alpha \wedge(d \alpha)^{n-1}=0, \quad X(0) \neq 0,
$$

meets $\tilde{S}$ transversally at 0 . Hence $X$ also meets $S_{2 n-1}$ transversally at 0 . Therefore by means of elements from $G_{S}$ one can reduce $X$ to the form $\pm \frac{\partial}{\partial x_{1}}$. Thus $\tilde{S}$ is locally a graph 
of a smooth function $\theta:\left(S_{2 n-1}, 0\right) \rightarrow(R, 0)$. Hence $\left(x_{2}, \ldots, x_{n}, \ldots, y_{1}, \ldots, y_{n}\right)$ define a coordinate system on $\tilde{S}$. From 2 and 3 it follows that $\iota^{*} \alpha$ satisfies the assumptions of Lemma 2.5. Therefore we have

$$
\phi^{*} \iota^{*} \alpha=d y_{1}+d y_{2}+\sum_{i=2}^{n} x_{i} d y_{i},
$$

where $\phi:(\tilde{S}, 0) \rightarrow(\tilde{S}, 0)$ is a diffeomorphism which preserves $\tilde{S} \cap\left\{y_{1}=0\right\}$. Let $\Phi \in G_{S}$ be such that

Hence we obtain

$$
\Phi(x, y)=\left(x_{1}, \phi\left(x_{2}, \ldots, x_{n}, y_{1}, \ldots, y_{n}\right)\right)
$$

$$
\iota^{*} \Phi^{*} \alpha=d y_{1}+d y_{2}+\sum_{i=2}^{n} x_{i} d y_{i}
$$

It is easy to check that the vector field $X$ satisfies the following conditions:

$$
X\rfloor \alpha=0 \quad \text { and } \quad X\rfloor d \alpha=\varphi \alpha,
$$

where $\varphi: R^{2 n} \rightarrow R$ is a smooth function. Thus by Lemma 2.3 , we obtain

$$
\alpha=h\left(d y_{1}+d y_{2}+\sum_{i=2}^{n} x_{i} d y_{i}\right),
$$

where $h: R^{n} \rightarrow R$ is a smooth function such that $\left.h\right|_{\tilde{S}}=1$. We have

$$
(d \alpha)^{n}=n ! h^{n-1} \frac{\partial h}{\partial x_{1}} \Omega .
$$

On the other hand, by Lemma $2.1, \omega^{n}= \pm\left(x_{1}-g\right) \Omega$. Hence $n ! h^{n-1} \frac{\partial h}{\partial x_{1}}= \pm\left(x_{1}-g\right)$, and

$$
\frac{\partial h^{n}}{\partial x_{1}}= \pm \frac{1}{(n-1) !}\left(x_{1}-g\right)
$$

with an extra condition $\left.h\right|_{\left\{x_{1}=g\right\}}=1$. Solving this equation we get

$$
h=\sqrt[n]{\frac{ \pm 1}{2(n-1) !}\left(x_{1}-g\right)^{2}+1} .
$$

By the diffeomorphism $\Lambda^{-1} \in G_{\Sigma}$, where

$$
\Lambda(x, y)=\left(x_{1}, h(x, y) x_{2}, \ldots, h(x, y) x_{n}, y_{1}, \ldots, y_{n}\right)
$$

we reduce $\alpha$ to

The diffeomorphism

$$
\alpha=h\left(d y_{1}+d y_{2}\right)+\sum_{i=2}^{n} x_{i} d y_{i}
$$

$$
\Upsilon(x, y)=\left(\left(x_{1}-\zeta\right) \sqrt{(n-1) ! \sum_{i=0}^{n-1}\left(\begin{array}{c}
n \\
i+1
\end{array}\right)\left(\frac{ \pm\left(x_{1}-\zeta\right)^{2}}{2}\right)^{i}}-g, y_{1}, \ldots, x_{n}, y_{n}\right),
$$

where $\zeta$ is a function which does not depend on $x_{1}$ and satisfies

$$
\sqrt[n]{\frac{ \pm 1}{2(n-1) !} g^{2}+1}= \pm \frac{\zeta^{2}}{2}+1
$$


preserves the sets $S_{2 n-1}, S_{2 n-2}$ and

$$
\Upsilon^{*} \alpha=\left( \pm \frac{\left(x_{1}-\zeta\right)^{2}}{2}+1\right)\left(d y_{1}+d y_{2}\right)+\sum_{i=2}^{n} x_{i} d y_{i}
$$

If $\Upsilon$ does not belong to $G_{\Sigma}$ then we replace it by $\Theta \circ \Upsilon$, where

$$
\Theta(x, y)=\left(-x_{1}, x_{2}, \ldots, x_{n}, y_{1}, \ldots, y_{n}\right) .
$$

Hence we obtain

$$
\alpha=\left(1 \pm \frac{1}{2}\left(x_{1}-\psi\right)^{2}\right)\left(d y_{1}+d y_{2}\right)+\sum_{i=2}^{n} x_{i} d y_{i}
$$

Therefore

$$
\omega=d \alpha= \pm\left(x_{1}-\psi\right) d\left(x_{1}-\psi\right) \wedge d y_{1}+d\left(x_{2} \pm \frac{1}{2}\left(x_{1}-\psi\right)^{2}\right) \wedge d y_{2}+\sum_{i=3}^{n} d x_{i} \wedge d y_{i} .
$$

Finally, by means of $\Xi \in G_{\Sigma}$, where

$$
\Xi(x, y)=\left(x_{1}, x_{2} \pm \frac{1}{2}\left(x_{1}-\psi\right)^{2}, x_{3}, \ldots, x_{n}, \pm y_{1}, y_{2}, y_{3}, \ldots, y_{n}\right),
$$

we reduce $\omega$ to the form 7 .

Now we pass to the investigation of stability properties of symplectic structures on $S$.

Definition 2.2. Let $\omega$ be a symplectic form on $S$. Then $\omega$ is stable at $p \in S_{2 n-2}$ if for any neighbourhood $U$ of $p$ in $S_{2 n-2}$ there is a neighbourhood $V$ of $\omega$ (in the $C^{\infty}$ topology on closed 2-forms) such that if $\beta$ is in $V$, then there is a point $q \in U$ and a germ of a diffeomorphism $\Phi:\left(R^{2 n}, q\right) \rightarrow\left(R^{2 n}, p\right)$ which preserves $S$ and $\Phi^{*} \beta=\omega$.

It is easy to see that the Darboux form on $S$ is stable.

Proposition 2.3. Let $\omega$ be a symplectic structure on $S$. Assume $f(0)=0$ and $d f_{0} \neq 0$. Then $\omega$ is not stable at 0 .

Proof. From Theorem 2.1 it follows that $\omega$ can be reduced to the form

$$
\left(x_{1}-\psi\right) d\left(x_{1}-\psi\right) \wedge d y_{1}+\sum_{i=2}^{n} d x_{i} \wedge d y_{i} .
$$

Suppose the proposition is false. Let $U$ be a neigbourhood of $0 \in R^{2 n} \cdot \psi(0)=0 \in R$ is a critical value of $\left.\psi\right|_{U}$. From the Sard theorem we see that there is $\epsilon \in R$ which is not a critical value of $\left.\psi\right|_{U}$, in any neighbourhood of $0 \in R$. Let $\beta=\alpha+\epsilon d\left(x_{1}-\psi\right) \wedge d y_{1}$. Then we can find a diffeomorphism $\Phi$ which preserves $S$ and $\Phi^{*} \beta=\omega$. Hence

$$
\Phi^{*} \beta^{n}=\Phi^{*}\left(\left(x_{1}-\psi+\epsilon\right) \Omega\right)=\omega^{n}=\left(x_{1}-\psi\right) \Omega .
$$

Since $\Sigma_{2}(\omega)$ is tangent to $S_{2 n-1}$ at $0, \Sigma_{2}(\beta)$ is tangent to $S_{2 n-1}$ at $q=\Phi(0) \in S_{2 n-2}$. Therefore, we obtain

$$
\psi(q)=\epsilon, \quad d \psi_{q}=0,
$$

which contradicts the fact that $\epsilon$ is not a critical value of $\left.\psi\right|_{U}$. 
2.1. Remark. Let us consider the following semialgebraic set:

$$
\begin{gathered}
S=S_{2 n} \cup S_{2 n-2} \subset R^{2 n} ; \\
S_{2 n}=\left\{(x, y) \in R^{2 n}: x_{1}^{3}>y_{1}^{2}\right\}, \quad S_{2 n-2}=\left\{(x, y) \in R^{2 n}: x_{1}=0, y_{1}=0\right\} .
\end{gathered}
$$

We notice the difference with the previous space: $\partial S_{2 n}$ is a singular set.

We endow $S$ with a symplectic structure $\omega$. As before $G_{S}$ denotes the group of diffeomorphisms $\left(R^{2 n}, 0\right) \rightarrow\left(R^{2 n}, 0\right)$ preserving $S$. Let $\omega_{1}, \omega_{2}$ be two symplectic structures on $S$. We say that $\omega_{1}$ and $\omega_{2}$ are $S$-equivalent if and only if $\Phi^{*} \omega_{1}=\omega_{2}$ for some $\Phi \in G_{S}$. Now we can show the following

Proposition 2.4. Let $\omega$ be a symplectic structure on $S$. Assume $f(0)=0$ and $d f_{0} \neq 0$. Then $\omega$ is a singular form of type $\Sigma_{2,0}$ at zero.

Proof. By straightforward use of the proof of Proposition 2.1.

An analogous Darboux theorem for the space $S$ is proved by Arnold ([2]): Let $\omega$ be a symplectic structure on $R^{2 n}$. Then $\omega$ is $S$-equivalent with respect to formal equivalence to the Darboux form:

$$
\omega \sim \sum_{i=1}^{n} d x_{i} \wedge d y_{i}
$$

\section{References}

[1] R. Abraham and J. Marsden, Foundations of Mechanics, 2nd ed., Benjamin/Cummings, Reading, 1978.

[2] V. I. Arnold, Lagrangian manifolds with singularities, asymptotic rays and the open swallow tail, Functional Anal. Appl. 15 (1981), 235-246.

[3] M. Golubitsky and D. Tischler, An example of moduli for singular symplectic forms, Invent. Math. 38 (1977), 219-225.

[4] S. Janeczko, Coisotropic varieties and their generating families, Ann. Inst. H. Poincaré 56 (1992), 429-441.

[5] E. Lerman, R. Montgomery and R. Sjamaar, Examples of singular reduction, preprint, 1991.

[6] J. Martinet, Sur les singularités des formes différentielles, Ann. Inst. Fourier (Grenoble) 20 (1) (1970), 95-178.

[7] - Singularities of Smooth Functions and Maps, Cambridge Univ. Press, Cambridge, 1982.

[8] J. Moser, On volume elements on manifolds, Trans. Amer. Math. Soc. 120 (1965), 280-296.

[9] R. Sjamaar and E. Lerman, Stratified symplectic spaces and reduction, Ann. of Math. 134 (1991), 375-442.

[10] V. Guillemin and S. Sternberg, Symplectic Techniques in Physics, Cambridge Univ. Press, Cambridge, 1984.

[11] A. Weinstein, Lectures on Symplectic Manifolds, CBMS Regional Conf. Ser. in Math. 29, Amer. Math. Soc., Providence, R.I., 1977. 